\title{
Refractive index correction in optical coherence tomography images of multilayer tissues
}

Zahra Turani

Emad Fatemizadeh

Qiuyun $\mathrm{Xu}$

Steven Daveluy

Darius Mehregan

Mohammadreza Nasiriavanaki 


\section{Refractive index correction in optical coherence tomography images of multilayer tissues}

\author{
Zahra Turani, ${ }^{\text {a,b }}$ Emad Fatemizadeh, ${ }^{a}$ \\ Qiuyun Xu, ${ }^{\text {b }}$ Steven Daveluy, ${ }^{\text {c,d }}$ Darius Mehregan, ${ }^{c}$ \\ and Mohammadreza Nasiriavanaki ${ }^{\mathbf{b}, \mathbf{c}, \mathrm{d}, *}$ \\ aSharif University of Technology, Department of Electrical \\ Engineering, Tehran, Iran \\ bWayne State University, Department of Biomedical Engineering, \\ Detroit, Michigan, United States \\ 'College of Engineering and School of Medicine Wayne State \\ University, Department of Dermatology, Detroit, Michigan, \\ United States \\ ${ }^{\mathrm{d} C o l l e g e}$ of Engineering and School of Medicine Wayne State \\ University, Karmanos Cancer institute, Detroit, Michigan, \\ United States
}

Abstract. We propose an algorithm to compensate for the refractive index error in the optical coherence tomography (OCT) images of multilayer tissues, such as skin. The performance of the proposed method has been evaluated on one- and two-layer solid phantoms, as well as the skin of rat paw. @ 2018 Society of Photo-Optical Instrumentation Engineers (SPIE) [DOI: 10.1117/1.JBO.23.7.070501]

Keywords: optical coherence tomography; refractive index correction; skin; dermatology.

Paper 180121LR received Mar. 2, 2018; accepted for publication Jun. 7, 2018; published online Jul. 10, 2018.

\section{Introduction}

Optical coherence tomography (OCT) is a high-resolution, noninvasive imaging modality with a large number of applications in the fields of ophthalmology and dermatology. ${ }^{1-3}$ Light beams interact with microstructures in a complex tissue based on their optical properties. The beams entering the tissue, experience refraction, reflection, scattering, and absorption. The difference in the refractive indices of regions in a tissue makes the light beam refract at the boundaries. Thus, knowing the refractive indices of the layers is important to accurately reconstruct the image of the tissue. ${ }^{4}$ In practice, for image reconstruction, an approximated constant refractive index is considered for the entire tissue. As a result, pixel locations in the image are misplaced. This consequently misleads physicians regarding diagnostically important morphological features in the OCT image, e.g., location of different structures and thickness of layers. ${ }^{5}$

Various methods have been developed to elucidate the refractive indices. ${ }^{4,-8}$ Refractometry and ellipsometry are two popular

*Address all correspondence to: Mohammadreza Nasiriavanaki, E-mail: mrn .avanaki@wayne.edu methods for the refractive index measurement. Refractometry only obtains the deflection angle on the surface of the medium and cannot be used for multilayer tissues. In ellipsometry, the refractive index is measured based on the change of the light polarization during transmission and reflection, and compared with a predefined model. Ellipsometry is only used for very thin layers. ${ }^{6,9}$

The refractive indices of a tissue can be obtained using either hardware modification or postprocessing. Although hardware modification is considered the most optimum solution for refractive index correction in multilayer samples, it may require substantial hardware modification of the OCT system. ${ }^{10}$ Consequently, the modified OCT system would need recertification; a long regulatory process. This recertification process is usually not preferred by clinicians or manufacturers of clinical OCT systems.

Most of the current postprocessing methods are based on Snell's law. ${ }^{4,8}$ To use the Snell's law, the beam angles, before and after deflection, must be known. This methodology works well for samples with one or two layers. However, when the number of layers is more than two, e.g., in skin, the technique does not work efficiently. This is mainly due to the fact that Snell's law is a hierarchical process in which the refractive index should be corrected layer by layer. Moreover, this technique is time consuming, and its performance becomes limited as depth increases. The inverse refractive index correction algorithm is another postprocessing method used for the correction of refractive indices in the images of one-layer samples, e.g., bone. ${ }^{4}$

The ability of OCT to identify skin abnormalities is mainly based on the tissue morphology. Therefore, refractive index correction in OCT images of skin becomes of paramount importance. Considering the refractive index of the epidermis equal to $1.4,{ }^{11}$ and the refractive index used in the OCT image reconstruction equal to $1.3,{ }^{4}$ the epidermal thickness alters $>15 \% .^{12}$ This inaccuracy becomes more important and critical when it is used for tumor border detection or thickness measurement, especially at cosmetically sensitive regions, such as the face.

In this paper, we proposed a refractive index correction algorithm that can find and correct the refractive index of all layers in a multilayer tissue, simultaneously. This algorithm works in parallel and is not hierarchical. As layers are processed simultaneously, independent of how many tissue layers there are, the speed of the processing does not change significantly. In addition, speckle cannot affect the results of refractive index correction so there is no need for extra preprocessing required for speckle reduction.

\section{Materials and Methods}

The OCT machine used in this study is a swept-source OCT (SS-OCT) from Michelson Diagnostic ${ }^{\mathrm{TM}}$ (Vivosight, United Kingdom).

The OCT machine acquired images of one-layer and two-layer phantoms as well as rat paw skin. The one-layer phantom was constructed using $0.1 \% \mathrm{TiO}_{2}$ in the polyurethane (WC-781, BJB Enterprise Co.). The two-layer phantoms have one layer of $0.05 \%$ $\mathrm{TiO}_{2}$ in the polyurethane and one layer of the agar gelatin on the top. A 26 gage needle (NIPRO HYPODERMIC Dispensing) was manually inserted into the samples. The prepared OCT images were used for the evaluation of the proposed refractive index correction algorithm [see Fig. 1(a) as an example].

In the OCT system, the reflected beams from sample and reference arms are interfered and recorded at a photodetector

$1083-3668 / 2018 / \$ 25.00$ (C) 2018 SPIE 


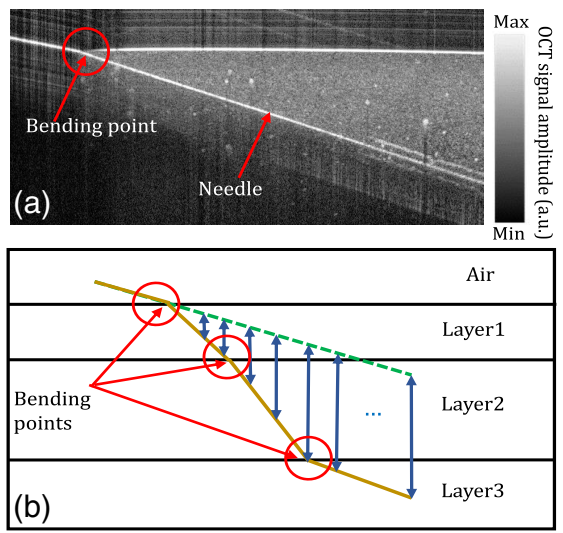

Fig. 1 (a) OCT image of one-layer polyurethane phantom. Bending points are where the refractive index/speed of light in tissue is altered. (b) Schematic of refraction and the impact of inaccurate refractive index in the reconstruction of a three-layer phantom with an inserted needle. Yellow solid line: image of the needle, green dashed line: refractive index corrected image of the needle.

$\left[I_{\text {Measured }}(t)\right]$. The image intensity at the depth $Z,\left(I_{Z}\right)$, is equal to $I_{\text {Measured }}$ at the time $t_{Z}=t_{0: z_{1}}+t_{z_{1}}:\left(z_{1}+z_{2}\right)+\ldots+$ $t_{\left(z_{1}+\ldots+z_{i-1}\right):(Z)}$ (e.g., in the three-layer example of Fig. 1(b), if the thickness of layer 1 is $z_{1}=0.3$, the thickness of layer 2 is $z_{2}=0.6$, and the thickness of layer 3 is $z_{3}=0.4, t_{Z}$ at the depth of $Z=1.3$ would be $t_{Z}=t_{0: 0.3}+t_{0.3: 0.9}+t_{0.9: 1.3}$, where $z_{i}$ is the thickness of the layer $i$ [Eqs. (2) and (3)]

$Z=\frac{1}{2} \mathrm{SL} \cdot t_{Z}$,

$I_{Z}=I_{\text {Measured }}\left(t_{Z}\right)$

where SL $\propto \frac{1}{\mathrm{RI}}$ is the speed of the light, and RI is the predefined refractive index of the corresponding medium, used by the OCT system. Equations (2) and (3) show that the travel time depends on the layer thicknesses and the refractive indices. For an accurate image reconstruction, it is necessary to have the exact value of the speed of light or the refractive index at each layer of a complex tissue. In the image reconstruction algorithm of OCT, this dependency is not considered, and only a constant refractive index is assigned to the entire tissue. In Fig. 1(b), a scenario is demonstrated where a needle is inserted into a multilayer tissue and appears bent at multiple interfaces. In this figure, the corrected image of the needle is also shown. Figure 1(a) shows an OCT B-scan image of a one-layer phantom with a metal needle inside. As the refractive index of the air and tissue is different, the needle appears bent at the interface of the air and tissue. It should be noted that it is expected to visualize the needle as a straight object in the image. To this end, the refractive index needs to be corrected for the tissue layers. The input of the algorithm is a fixed refractive index used for reconstruction in the OCT system, used as initial value in the algorithm, as well as the orientation of the needle in the OCT image. The outputs are the corrected refractive indices of the layers followed by the updated correct placement of the pixels and the thickness of the layers.

For refractive index correction of an OCT image of the skin, a needle penetrated into the skin is used. In the OCT image, first, the slope of the needle in the air is measured. Based on this, a hypothetical line is modeled within the tissue. By changing the refractive index of the layers and finding the new place of the pixels in each A-scan, the root mean square error (RMSE)
Table 1 Results of the hypothetical sample scenario shown in Fig. 1(b). A hypothetical value of refractive index and layer thickness before correction is assigned to each layer of Fig. 1(b). The correct refractive index and layer thickness is calculated using the proposed method. The percentage of the thickness change for each layer can be seen in the right column.

\begin{tabular}{|c|c|c|c|c|c|}
\hline \multirow[b]{2}{*}{ Layer } & \multicolumn{2}{|c|}{$\begin{array}{l}\text { Before refractive } \\
\text { index correction }\end{array}$} & \multicolumn{2}{|c|}{$\begin{array}{l}\text { After refractive } \\
\text { index correction }\end{array}$} & \multirow{2}{*}{$\begin{array}{l}\% \text { Thickness } \\
\text { change after } \\
\text { correction (\%) }\end{array}$} \\
\hline & $\begin{array}{l}\text { Thickness } \\
{[\mathrm{mm}]}\end{array}$ & $\begin{array}{l}\text { Refractive } \\
\text { index }\end{array}$ & $\begin{array}{l}\text { Thickness } \\
{[\mathrm{mm}]}\end{array}$ & $\begin{array}{c}\text { Refractive } \\
\text { index }\end{array}$ & \\
\hline 1 & 0.30 & 1.3 & 0.24 & 1.6 & 20 \\
\hline 2 & 0.60 & 1.3 & 0.41 & 1.9 & 32 \\
\hline 3 & 0.40 & 1.3 & 0.35 & 1.5 & 13 \\
\hline
\end{tabular}

between the hypothetical line and the line of the needle over the A-scans is calculated. The values of RMSE show the average of distance (in pixels) between the needle position and its correct position in all A-scans. A smaller value of RMSE means a more accurate refractive index of the layers. The calculated refractive index values that minimize the RMSE are the true refractive indices of the layers. The corresponding pixel position and refractive indices related to the minimum RMSE are the final updated pixel position and refractive indices. This means that the location of the layers boundaries would be updated as well as all pixels within the layers. In Fig. 1(b), a scenario is depicted where the image of the needle in a multilayer synthetic sample is corrected. Table 1 shows the variations in thicknesses and refractive indices of different layers before and after refractive index correction by the proposed algorithm; i.e., a hypothetical value for refractive index and layer thickness is assigned to each layer of Fig. 1(b), then the correct refractive index and layer thickness are calculated using the proposed method and shown in Table 1. Also, the percentage of the thickness change for each layer can be seen in Table 1. The pseudocode of the algorithm is given below (Algorithm 1).

\section{Experimental Results and Discussion}

The proposed algorithm was implemented in MATLAB 2015 in a computer with a core ${ }^{\mathrm{TM}}$ i7 CPU and 8 GB memory. The performance of the proposed method was evaluated using one- and two-layer solid phantoms, as well as rat paw skin. For each sample, 50 OCT B-scan images were acquired from different transversal locations.

The results of the refractive index correction for one- and two-layer phantoms and rat skin are shown in Fig. 2. The needle bends in the original images [Figs. 2(a), 2(c), and 2(e)] were corrected after applying the proposed algorithm [Figs. 2(b), 2(d), and 2(f)]. The value of the refractive index (before and after correction), RMSE, and the processing time for the corrections are listed in Table 2. The presented result for each sample is the average of values obtained from 50 images. An equivalence test was applied to each pair of images and $p$ values were calculated for all pairs in each dataset to evaluate the similarity between the RIs of each layer. The calculated $p$ value for the measurement of the refractive index of each layer is $<0.05$ with $\Delta=0.03$ for the phantoms and real tissue. It means that the probability of the difference between the refractive indices of the corresponding layers of each pair of images of a dataset is $>0.03$, is $<0.05$, indicating that there is no significant difference 
Algorithm 1 Pseudo code of the proposed method.

Initializing

$c$ number of pixels over each column of image

$\mathrm{SL}_{i}$ speed of light at layer $i$ of sample

$\mathrm{RI}_{i}$ refractive index at layer $i$ of sample

$z_{i}$ thickness of layer $i$.

$n$ the number of pixels in the image that need replacement,

$P_{\text {hypo }}$ a vector of hypothetic location of the needle along its slope in the air.

$P_{\mathrm{RI}-\text { corr }}$ a vector of refractive index corrected pixel locations.

SL constant speed of light used by OCT imaging system

$Z_{o}$ original depth calculated by OCT system using incorrect SL

1. Layer segmentation*

1.1. In each A-scan, find the boundary between air and sample.

1.2. In each A-scan, find the boundaries between layers of sample.

2. Find refractive index of layers

2.1. The slope of the needle in air is calculated. The needle in air is assumed to be continued into the tissue with the same slope [Fig. 1(b)].

2.2. Each A-scan in the image is temporally modified for each depth, $Z_{k}=z_{1}+z_{2}+\ldots+z_{i}, k=1: c$ such as below:

2.2.1. Initialize: $R I_{1}=R I_{2}=\ldots=R I_{i}=1.35$,

2.2.2. For each pixel, find the time of detecting the intensity value at depth $Z_{k},\left(t_{k}\right)$, using the following equation:

$$
\begin{aligned}
& t_{k}=\left(\frac{z_{1}}{S L_{1}}+\frac{z_{2}}{S L_{2}}+\ldots+\frac{z_{i}}{S L_{i}}\right) \times 2, \\
& \mathrm{SL}_{i}=w \frac{1}{R I_{i}}, \quad w=1,
\end{aligned}
$$

where $w$ is a constant coefficient.

2.2.3. Find the correct intensity value of depth $Z_{K},\left(I_{Z_{K}}\right)$, from the original image with below equations:

$$
\begin{aligned}
& Z_{o}=\frac{1}{2} \text { SL. } t_{Z}, \quad t_{Z}=t_{k} \\
& I_{Z_{K}}=I\left(Z_{o}\right)
\end{aligned}
$$

2.2.4. Go to 2.2.1 and do steps 2.2.1 to 2.2.3 for all A-scans and reconstruct the needle with its new placement.

2.2.5. Measure the Euclidean distance between the hypothetical needle and the RI corrected needle in the image by considering RMSE criteria.

$$
\mathrm{RMSE}=\sqrt{\frac{1}{n} \sum_{I=1}^{n}\left[P_{\mathrm{hypo}}(I)-P_{\mathrm{RI}-\mathrm{corr}}(I)\right]^{2}}
$$

2.2.6. Change $\mathrm{RI}_{i} \mathrm{~s}$ and repeat steps 2.2 .2 to 2.2 .5 to minimize the RMSE.

3. Update the position of pixels and reconstruct the OCT image with the new Rls. Each pixel locates in its correct position when the RMSE is minimized.

* for the segmentation of the layers, the method proposed in Ref. 5 is used.

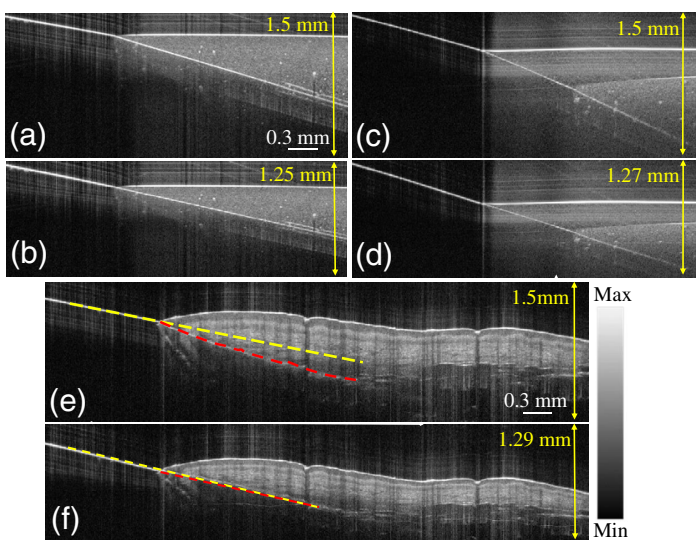

Fig. 2 Refractive index correction results. The yellow value at the top of each image shows the thickness of that image. (a) The original OCT image of one-layer polyurethane phantom, (b) refractive index corrected OCT image of one-layer polyurethane phantom, (c) original OCT image of two-layer polyurethane phantom, (d) refractive index-corrected OCT image of two-layer polyurethane phantom, (e) original OCT image of rat paw skin, and (f) refractive index corrected OCT image of rat paw skin.

\begin{tabular}{|c|c|c|c|c|c|}
\hline \multirow[b]{2}{*}{ Sample } & & \multirow{2}{*}{$\begin{array}{l}\text { Refractive } \\
\text { index }\end{array}$} & \multirow{2}{*}{$\begin{array}{c}\text { Processing } \\
\text { time }(s)\end{array}$} & \multicolumn{2}{|c|}{ RMSE (pixel) } \\
\hline & & & & Before & After \\
\hline \multicolumn{2}{|c|}{ One-layer phantom } & $1.30 \pm 0.03^{*}$ & 0.41 & 42.9 & 3.3 \\
\hline \multirow{2}{*}{$\begin{array}{l}\text { Two-layer } \\
\text { phantom }\end{array}$} & layer1 & $1.16 \pm 0.03^{*}$ & 0.66 & 44.6 & 5.4 \\
\hline & layer2 & $1.32 \pm 0.04^{*}$ & & & \\
\hline \multirow[t]{5}{*}{ Rat skin } & SC & $1.30 \pm 0.04^{*}$ & 1.70 & 40.1 & 4.9 \\
\hline & EP & $1.39 \pm 0.04^{*}$ & & & \\
\hline & DEJ & $1.25 \pm 0.03^{*}$ & & & \\
\hline & $\mathrm{DE}$ & $1.13 \pm 0.05^{\star}$ & & & \\
\hline & $\begin{array}{l}\text { Deeper } \\
\text { layers }\end{array}$ & $1.19 \pm 0.07^{*}$ & & & \\
\hline
\end{tabular}
In (e) and (f), the yellow line indicates the hypothetical needle and the red line shows the place of the needle in the tissue.

Table 2 Results of refractive index correction for images of phantom and rat skin in Fig. 2.

Note: SC, stratum corneum; EP, epidermis; DE, dermis; DEJ, dermoepidermal junction.

$p<0.05$.

between the RI of the same layers in these 50 locations/images of the rat paw skin as well as two phantom datasets. According to Eq. (2) in Ref. 13, the refractive index of rat paw skin obtained from the proposed method is calculated correctly. Figure 2 shows that the imaging depth is decreased about $15 \%$ after the refractive index correction.

In Fig. 3, the changes in the thickness of the rat paw skin layers resulting from the correction algorithm are shown with more detail. We analyzed the changes in 50 images of rat skin, and the results of two sample images are shown in Fig. 3. The results are as follows: $16 \%$ thickness reduction in the epidermal thickness (ET) and 20\% reduction for the entire tissue thickness. The other observation was needle curvature in deeper levels of skin tissue [see Fig. 2(e)], this is due to the assumption that the 


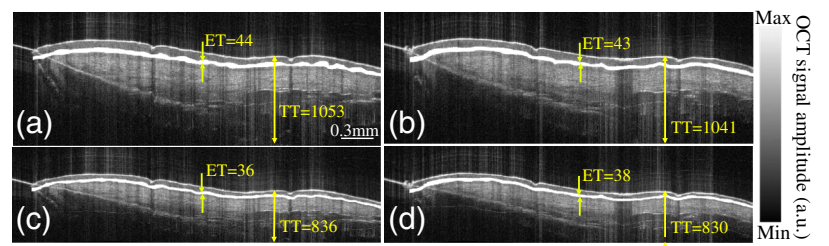

Fig. 3 (a) and (b) Two OCT B-scan images of a rat paw skin, (c) and (d) corresponding B-scan images of the corrected refractive index. The yellow arrows show the thickness of the epidermis and the entire tissue before and after the refractive index correction. ET, epidermal thickness; TT, total skin thickness. All the values in this figure are in micrometer.

refractive index is constant in the entire image, when it is not. Compared with the methods that are based on Snell's law, our proposed method is more feasible for OCT images of multilayer tissues [see Fig. 2(f)]. For single-layer and two-layer phantoms, where the correction methods that work based on Snell's law perform effectively, the results of the proposed method and Snell's law methods are comparable for one-layer phantom, the refractive index obtained 1.19 while it is 1.17 using Snell's law. For two-layer phantom, the refractive index of layer one and layer two are obtained 1.16 and 1.32 respectively, while they are 1.14 and 1.29 using Snell's law. We expect this because Snell's law has a good performance in simple one- and twolayer samples. It should be noted that for finding the RI using Snell's law, it is necessary to know the angle of each deflection. Thus, with additional layers of tissue, applicability of Snell's law decreases while the proposed method can apply to the tissues independent of how many layers there are.

The processing time for the correction of the refractive indices in the proposed method is also calculated. In most cases, it is less than one second, which indicates the feasibility of implementation of this method in OCT software. In this way, when the OCT image is acquired, the refractive index correction is automatically applied, and a refractive index corrected image is shown on the screen. As diagnosis via OCT is based on layer detection and skin layer thickness measurement in dermatology, such a correction is of importance. One requirement of the proposed method is knowing a particular reference structure, for which a needle inserted into the skin is used.

The proposed method is specifically designed for OCT imaging of skin where other refractive index correction methods cannot be easily applied. Requiring the insertion of a needle into the skin does add a slightly invasive component to the imaging procedure. However, the main application of the introduced technique is to improve the accuracy of the skin thickness/depth measurement. Measurement accuracy is of critical importance when diagnosing or planning procedures to treat skin tumors or abnormalities. Needle insertion to induce analgesia in the skin is a necessary step prior to any diagnostic or therapeutic procedure. Therefore, the needle insertion can serve two purposes: refractive index correction and preprocedure analgesia. This will not actually contribute any additional pain or risk to the procedure overall. One important application of our method is accurate measurement of the Breslow depth in melanoma, which is used to stage the tumor and acts as the single most important determinant of the prognosis and treatment plan. The proposed method corrects the thickness in each A-scan separately. Therefore, in the case of having 3-D OCT volume instead of 2-D image, the proposed method is also applicable (using voxels instead of pixels).

\section{Conclusion}

In this study, we proposed a method for refractive index correction in OCT images of skin. This method uses the location of the pixels and modifies them over each A-scan. In addition, the pixel intensities are not used in the proposed method. Therefore, the presence of speckles in the OCT image does not affect the performance of the algorithm. The algorithm is computationally inexpensive and can be applied to heterogeneous tissues with an anisotropic nature. The proposed method does not need any prior information, such as the deflection angles or boundary layer location. The results show that the refractive index correction has significantly improved the accuracy of the geometric features of the needle, indicating that the anatomical features of the skin are more accurate. The statistical analysis of the results demonstrates that the refractive indices obtained for the skin layers can be used for the nearby skin. The method has many potential benefits in dermatology.

\section{Disclosures}

The authors have no relevant financial interests in the letter and no other potential conflicts of interest to disclose.

\section{Acknowledgments}

This project has been funded by Institutional Research Grant No. 14-238-04-IRG from the American Cancer Society, Michelson Diagnostics, and Wayne State University Startup fund.

\section{References}

1. Q. Xu et al., "Swept-source optical coherence tomography-supervised biopsy," Dermatol. Surg. 44, 768-775 (2018).

2. S. Adabi et al., "An overview of methods to mitigate artifacts in optical coherence tomography imaging of the skin," Skin Res. Technol. 24, 265-273 (2018)

3. S. Adabi et al., "Universal in vivo textural model for human skin based on optical coherence tomograms," Sci. Rep. 7, 17912 (2017).

4. J. Stritzel, M. Rahlves, and B. Roth, "Refractive-index measurement and inverse correction using optical coherence tomography," Opt. Lett. 40, 5558-5561 (2015).

5. M. R. Avanaki and A. Hojjatoleslami, "Skin layer detection of optical coherence tomography images," Opt. Int. J. Light Electron. Opt. 124, 5665-5668 (2013).

6. J. Charmet and P. De Gennes, "Ellipsometric formulas for an inhomogeneous layer with arbitrary refractive-index profile," J. Opt. Soc. Am. 73, 1777-1784 (1983).

7. A. Knu and M. Boehlau-Godau, "Spatially confined and temporally resolved refractive index and scattering evaluation in human skin performed with optical coherence tomography," J. Biomed. Opt. 5, 83-92 (2000).

8. F. N. Golabchi, "Refractive effects on optical measurement of alveolar volume: a 2-D ray-tracing approach," in Annual Int. Conf. of the IEEE Engineering in Medicine and Biology Society, pp. 7771-7774. (2011).

9. P. H. Tomlins, "Optical coherence refractometry," Opt. Lett. 33, 22722274 (2008).

10. G. Tearney et al., "Determination of the refractive index of highly scattering human tissue by optical coherence tomography," Opt. Lett. 20, 2258-2260 (1995).

11. J. Welzel, "Optical coherence tomography in dermatology: a review," Skin Res. Technol. 7, 1-9 (2001).

12. M. Mogensen, "Morphology and epidermal thickness of normal skin imaged by optical coherence tomography," Dermatology 217, 14-20 (2008).

13. A. N. Bashkatov, "In-vivo and in-vitro study of control of rat skin optical properties by action of osmotical liquid," in Optics and Optoelectronic Inspection and Control: Techniques, Applications, and Instruments, pp. 300-311 (2000). 